\title{
Molecular detection of SHV-Type ESBL in E. coli and $K$.pneumoniae and their antimicrobial resistance profile
}

\section{Sayran Hamad Haji* Salah Tofik J alal** Sharmin Abdullah Omer* Ahang Hasan Mawlood**}

\section{Abstract}

Background and objective: The increase in the incidence of Beta-lactam resistance in Gram-negative bacteria has become a main clinical problem worldwide that limits therapeutic options. The production of extended-spectrum blactamases is the major source of resistance to newer beta-lactam antibiotics in Enterobacteriaceae. This study aimed to check the presence of extended-spectrum $\beta$-lactamase producing $K$. pneumoniae and $E$. coli and the evaluation of ESBL among those isolates.

Methods: A total of $120 \mathrm{E}$. coli and K. pneumoniae clinical isolates were obtained from Rizgary Teaching Hospital in Erbil City, Iraqi Kurdistan Region, during the first six months of 2016. All isolates were identified and checked for the production of ESBL using Vitek 2 automated system. The technique of PCR was performed for the detection of the presence of blaSHV gene from these isolates. The antibiotic resistant profiles for these isolates were also investigated.

Results: The overall proportion of ESBL-producing E. coli and $K$. pneumoniae isolates, $77(76.2 \%)$ and $15(78.9 \%)$ isolates were ESBL producers, respectively. ESBL-producing isolates were significantly more resistant than Non-ESBL-producers $(P<0.05)$. PCR performed on $30 \mathrm{ESBL}$ positive isolates, $21(70 \%)$ isolates were of $E$. coli, and $9(30 \%)$ isolates were of K.pneumoniae. The ESBL B-lactamase related SHV gene was detected only in $11(36.6 \%)$ isolates, $6(28.5 \%)$ of $E$. coli and $5(55.5 \%)$ of $K$. pneumoniae isolates respectively. All isolates were susceptible to carbapenems.

Conclusion: The great diversity of ESBL and the prevalences of clinical isolates of $E$. coli and $K$. pneumoniae producing these enzymes indicate that this is an important problem in our region. The most active antimicrobial agent against isolates used in this study was carbapenem. It is, therefore strongly recommended to consider carbapenems as the drug of choice for such multi-drug resistant ESBL-producing microorganisms.

Keywords: ESBL; blaSHV gene; E. coli; K. pneumoniae.

\section{Introduction}

The incidence of resistance to antimicrobial agents has been a prime public health issue over the last decade, that influencing the monitoring and treatment of infections in nosocomial and health care-associated settings, and in the community. ${ }^{1}$ Specifically, the emergence of extendedspectrum $\beta$-lactamase (ESBL) - producing bacteria such as $E$. coli and $K$. pneumoniae as the most prevalent ESBL producing bacterial that become major causative agents for increasing resistance to $\beta$-lactam antibiotics. ${ }^{2}$ Because $\beta$-lactam antimicrobial agents are among the most widely used antibiotics to treat those infections. $^{3,4}$ All ESBL producers confer resistance to all generations of cephalosporins, penicillins, and aztreonam (except for cephamycins or carbapenems) via hydrolysis of these antibiotics. The inactivation is done by antibiotic combination therapy using B-lactamase inhibitors with antibiotics such as clavulanic acid, sulbactam, or tazobactam. ${ }^{2,5}$ Production of ESBL enzymes is an

* Department of Pharmacognosy, College of Pharmacy, Hawler Medical University, Erbil, I raq.

** Department of Medical Microbiology, College of Health Science, Hawler Medical University, Erbil, I raq. 
important mechanism of B-lactam resistance in Enterobacteriaceae. ${ }^{2}$ In addition, ESBL producing organisms display co-resistance to many other types of antibiotics resulting in limitation of treatment alternatives. ${ }^{6,7}$ The ESBL genes are mostly plasmid-encoded which can easily spread from one organism to another. ${ }^{8}$ ESBLs are grouped into four classes $A, B, C$ and $D$ enzymes and eight subgroups according to their functional and structural characteristics. Cefotaximase (CTX-M), temoneira (TEM) and sulfhydryl variable (SHV) are a class A $\beta$ lactamases. ${ }^{9}$ TEM and sulphydryl variable SHV are the most common types. However, CTX-M type is the major type in some countries. ${ }^{10}$ Many ESBLs arise because of mutations in the natural B-lactamase related genes like TEM-1, TEM-2, and SHV-1 genes. ${ }^{11}$ While these basic enzymes exist predominantly in Klebsiella species and E. coli. ${ }^{12}$ Until now, more than 400 different ESBL variants have been identified. ${ }^{13}$ Detection of ESBL is initially based on phenotypic tests, such as the double-disc synergy test and combined disc method. However, these tests are time-consuming and inhibited by the AmpC $\beta$-lactamases. Over the past years, PCR has replaced traditional phenotypic methods. ${ }^{6}$ Determination of ESBL genes, including TEM and SHV, by molecular techniques in ESBL producing bacteria and their pattern of antimicrobial resistance can provide reliable information about their epidemiology and risk factors related to their infections. ${ }^{13}$ Navon-Venezia and cowarkers ${ }^{14}$ indicated that these phenotypic tests need to be monitored periodically, as their performance may change with the introduction of the new enzyme by such bacteria. The present study aimed to estimate the prevalence of ESBL producing E. coli and K. pneumoniae with detection of related SHV genes by PCR among patients admitted for treatment at Rezgari Teaching Hospital in Erbil City. In addition to investigating the antimicrobial resistance profile of those ESBL producers.

\section{Methods}

\section{Study Design and Bacterial Isolates}

The present study was conducted at Rizgary Teaching Hospital in Erbil City, Iraqi Kurdistan Region, in which the prevalence of ESBL-producing E. coli and $K$. pneumoniae strains were evaluated over a period of 5 months. From January the $10^{\text {th }}$ to June the $20^{\text {th }} 2016$, a total of 120 consecutive non-repeated clinical isolates $(101 \mathrm{E}$. coli, and $19 \mathrm{~K}$. pneumoniae) were collected from different clinical specimens such as urine, tracheal aspirate, high vaginal swabs, wound swab and blood. The specimens were obtained by the clinical microbiology laboratory at a hospital as part of routine diagnostic activities. Isolates were identified to species level using standard microbiological methods. ${ }^{15}$ All isolates were stored at $-70^{\circ} \mathrm{C}$ in glycerol broth until tested. This project was approved at the first site by the Scientific and Research Ethics Committee at the College of Pharmacy, Hawler Medical University.

Identification and Characterization of Antimicrobial Susceptibility

In vitro susceptibility testing of all collected isolates to a wide range of antimicrobials was performed using VITEK 2 compact system, ${ }^{15}$ an automated ID and susceptibility (AST) system (bioMe'rieux, USA). VITEK 2 system includes an Advanced Expert System (AES) with a high sensitivity and specificity values $(94-100 \%)$ that considered rapid and reliable means for routine laboratory work that is widely used for identification and/or susceptibility testing. ${ }^{16} E$. coli and K. pneumoniae isolates were identified and tested for susceptibility profile by VITEK 2 system which usually uses different Antimicrobial Susceptibility Test cards (AST-cards) according to the expected pathogens. The related cards were inoculated and incubated in the machine according to the manufacturer's instructions. Vitek susceptibility test results were obtained as MIC values and shown as susceptible, intermediate or resistant 
according to National Committee for Clinical Laboratory Standard's breakpoint (National Committee for Clinical Laboratory Standards, 1999). ${ }^{15}$ Final results were interpreted using the Advanced Expert System (AES) (software version VT2R05.04). Testing was repeated wherever suggested by the AES. ${ }^{16,17}$

\section{Detection of ESBL by Phenotypic Method}

Each isolate was tested using the VITEK 2 system with the antimicrobial susceptibility test extend AST-EXN8 card. This system was designed to perform both screening and confirmatory tests for phenotypic detection of ESBL on the same plate. ${ }^{17}$ The test comprises a panel of six wells containing ceftazidime $0.5 \mathrm{mg} \mathrm{L}$, cefotaxime $0.5 \mathrm{mg} / \mathrm{L}$ and cefepime $1.0 \mathrm{mg}$ $\mathrm{L}$, the rest of three wells were filled with same three antibiotics in combination with clavulanic acid (4, 4 and $10 \mathrm{mg} \mathrm{L}$, respectively). Growth in each well was quantitatively assessed by means of an optical reader. The proportional reduction in growth in wells containing cephalosporin plus clavulanate compared with those containing the cephalosporin alone was considered to be indicative of ESBL production. All phenotypic interpretations of ESBLs were reported as a positive ESBL screening result. Strains were reported as ESBL-negative whenever phenotypic interpretations other than ESBLs were proposed by the AES. ${ }^{5,18}$

\section{Molecular Detection of ESBL Genes}

Conventional PCR was used for detection of the genes for SHV. Isolates sub-cultured from frozen stock using blood agar media. Cells were grown on blood agar plates for $24 \mathrm{~h}$ at $37^{\circ} \mathrm{C}$, and few colonies were resuspended in $500 \mathrm{ml}$ of sterile distilled water. The bacterial cells were lysed by heating at $95^{\circ} \mathrm{C}$ for $10 \mathrm{~min}$, and cellular debris was removed via centrifugation at 16,000 rpmfor $2 \mathrm{~min}$. The supernatant was used as the source of DNA template for PCR. ${ }^{19,20}$ About $5 \mu \mathrm{l}$ of DNA template was added to the final volume of $25 \mu \mathrm{l}$ Mastermix (GoTaq ${ }^{\circledR}$ Green Master Mix, a premixed ready-to-use solution containing Taq DNA polymerase, dNTPs, $\mathrm{MgCl}_{2}$, and reaction buffers at optimal concentrations for efficient amplification of DNA templates) GoTaq ${ }^{\circledR}$ Green Master Mix(Promega M7122, USA). Primers were used previously by others ${ }^{20}$ obtained from Integrated DNA Technology (IDT, Canada), used for blaSHV forward 5'TTAACTCCCTGTTAGCCA $3^{\prime}$ (TM $50.1^{\circ} \mathrm{C}$ ) and reverse 5' GATTTGCTGATTTCGCCC $3\left(\right.$ TM $\left.52.0^{\circ} \mathrm{C}\right)$ having product size $768 \mathrm{bp}^{20}$ with accession no. EF 035566.1 in the gene bank. These primers were added to make the final concentration of $1.0 \mu \mathrm{M}$. Amplification was performed in a Techne Genius Thermocycler, with cycling parameters comprising initial denaturation at $94^{\circ} \mathrm{C}$ for $3 \mathrm{~min}$ followed by 30 cycles each of denaturation at $94^{\circ} \mathrm{C}$ for $30 \mathrm{sec}$, annealing at $50^{\circ} \mathrm{C}$ for $30 \mathrm{sec}$, amplification at $72^{\circ} \mathrm{C}$ for two mins, and the final extension at $72^{\circ} \mathrm{C}$ for ten mins. The amplified products were separated in 1.2 percent agarose gel. The agarose gel was prepared by mixing $1.2 \mathrm{~g}$ agarose powder with $100 \mathrm{~mL}$ 1xTBE buffer in a microwavable flask. The mixture was dissolved in a microwave for a few mins then left at room temperature to cool down to about $50^{\circ} \mathrm{C}$. Ethidium bromide was then added to a final concentration of $0.5 \mathrm{mg} / \mathrm{ml}$. The agarose then poured into a gel tray with the good comb in place until it has completely solidified. A $100-1000$ bp DNA molecular weight marker (Norgen Biotech Corp Cat. No. 11600) was used to measure the bands of amplified products. PCR product samples and the ladder were then loaded into the wells of the gel starting with the ladder into the first well. No positive and negative controls were used because the product size was known $768 \mathrm{bp}$ and confirmed by measuring its size using the ladder as a reference. The gel was then run at a voltage of $100 \mathrm{~V}$ until the dye line was approximately $75-80 \%$ of the way down the gel and was visualized the DNA fragments using a UV light camera in a dark room. 


\section{Statistical Analysis}

The statistical package for the social science (SPSS 23 Chicago, IL, USA) was used to compare the mean value \pm standard deviations (SD) of antibiotic resistance. The frequencies, percentages, and Chi-Square were used in the study, and a $P$ value of $\leq 0.05$ was considered as statistically significant.

\section{Results}

In this investigation, isolates were identified up to species level by VITEK 2 automated microbiology system. Out of 120 collected isolates, 101 isolates were of $E$. coli, and 19 isolates were of $K$. pneumoniae. Of those $101 \mathrm{E}$. coli isolates and 19
K. pneumoniae isolates, $77(76.2 \%)$ and $15(78.9 \%)$ were ESBL producers, respectively (Table 1). The resistant pattern of120 E. coli and K. pneumoniae isolates to 16 antimicrobial agents included in AST-EXN8 card are shown in Table 2. All isolates $(100 \%)$ were susceptible to imipenem and ertapenem. The majority of isolates showed a high degree of resistance to ampicillin (\%91.6), cefazolin (\%80.8), ceftazidime alone with ceftriaxone and cefepime (\%79.1). Whereas, resistance to other antibiotic classes was moderate. Up to $60 \%$ of the isolates exhibited a multidrug-resistance (MDR) phenotype.

Table1: ESBL producing E. coli and K. pneumoniae isolates.

\begin{tabular}{lcccc}
\hline Pathogens & $\begin{array}{c}\text { No. of } \\
\text { isolates }\end{array}$ & $\begin{array}{c}\text { No of ESBL-producer } \\
(\mathbf{\%})\end{array}$ & $\begin{array}{c}\text { No. of Non-ESBL-producer } \\
\mathbf{( \% )}\end{array}$ & P value \\
\hline E.coli & 101 & $77(\% 76.2)$ & $24(\% 23.7)$ & \\
K. pneumoniae & 19 & $15(\% 78.9)$ & $4(\% 21.0)$ & 0.797 \\
Total & 120 & $92(\% 76.6)$ & $28(\% 23.3)$ & \\
\hline
\end{tabular}

Table 2: Number and percentage of antimicrobial resistant of both E.coli and K. pneumoniae isolates.

\begin{tabular}{lcccccc}
\hline \multirow{2}{*}{ Antibiotics } & \multicolumn{2}{c}{ E.coli } & \multicolumn{2}{c}{ Kll resistant isolates } \\
& \multicolumn{2}{c}{$\mathbf{n = 1 0 1}$} & \multicolumn{2}{c}{$\mathbf{n = 1 9}$} & \multicolumn{2}{c}{ Total } \\
& No. & $(\%)$ & No. & (\%) & No. & (\%) \\
\hline Ampicillin & 92 & $(91)$ & 18 & $(94.7)$ & 110 & $(91.6)$ \\
Cefazolin & 82 & $(81.1)$ & 15 & $(78.9)$ & 97 & $(80.8)$ \\
Ceftazidime & 80 & $(79.2)$ & 15 & $(78.9)$ & 95 & $(79.1)$ \\
Ceftriaxone & 80 & $(79.2)$ & 15 & $(78.9)$ & 95 & $(79.1)$ \\
Cefepime & 80 & $(79.2)$ & 15 & $(78.9)$ & 95 & $(79.1)$ \\
Ampicillin/Sulbactam & 59 & $(58.4)$ & 10 & $(52.6)$ & 69 & $(57.5)$ \\
Trimethoprim/Sulfamethoxazole & 53 & $(52.4)$ & 15 & $(78.9)$ & 68 & $(56.6)$ \\
Gentamicin & 56 & $(55.4)$ & 8 & $(42.1)$ & 64 & $(53.3)$ \\
Levofloxacin & 51 & $(50.4)$ & 6 & $(31.5)$ & 57 & $(47.5)$ \\
Ciprofloxacin & 49 & $(48.5)$ & 5 & $(26.3)$ & 54 & $(45)$ \\
Tobramycin & 37 & $(36.6)$ & 6 & $(31.5)$ & 43 & $(28.3)$ \\
Amoxicillin-Clavulanic acid & 27 & $(26.7)$ & 5 & $(26.3)$ & 32 & $(26.6)$ \\
Piperacillin/Tazobactam & 13 & $(12.8)$ & 5 & $(26.3)$ & 18 & $(15)$ \\
Nitrofurantion & 6 & $(5.9)$ & 3 & $(15.7)$ & 9 & $(7.5)$ \\
\% Multidrug resistance & 68 & $(67.3)$ & 11 & $(57.8)$ & 79 & $(65.8)$ \\
\hline
\end{tabular}


The mean and SD of antimicrobial resistance of $E$. coli and $K$. pneumoniae isolates (ESBL-producers and Non-ESBLproducers) are summarized in Table 3. ESBL-producing isolates showed the maximum rate of resistance to Ampicillin as well as oxyiminocephalosporins (100\%) and susceptible to Carbapenems (100\%). While the minimum resistance rate was seen with nitrofurantoin (5.4\%), Piperacillin/ Tazobactam (13\%), Amoxicillin-Clavulanic Acid (30.4\%) and Tobramycin (42.3\%). The Non ESBL-producing isolates showed high resistance rate to Ampicillin (64.2\%),
Trimethoprim/Sulfamethoxazole along with Levofloxacin (35.7\%), Ampicillin/ Sulbactam and Ciprofloxacin (32.1\%), while minimum rate of resistance was seen with oxyiminocephalosporins along with nitrofurantoin $(10.7 \%)$, and Amoxicillin-clavulanic acid (14.2\%), while no resistance was seen with carbapenems $(0 \%)$. ESBL-producing isolates were significantly more resistant than Non-ESBL -producers $(P<0.05)$. Multi-drug resistance (MDR) was higher among ESBL-producing $E$. coli and $K$. pneumoniae isolates than non ESBL-producing isolates in general.

Table 3: Mean and SD of antimicrobial resistant of both ESB and non-ESBL- producing E.coliand K. pneumoniae isolates.

\begin{tabular}{|c|c|c|c|}
\hline Antibiotics & $\begin{array}{c}\text { Non-ESBL-Producer } \\
\qquad n=28\end{array}$ & $\begin{array}{l}\text { ESBL-Producer } \\
\qquad n=92\end{array}$ & $P$ value \\
\hline Ampicillin & $64.2 \pm 0.018$ & $100 \pm 0.001$ & $<0.001$ \\
\hline Levofloxacin & $35.7 \pm 0.020$ & $51.0 \pm 0.020$ & $<0.001$ \\
\hline Trimethoprim/Sulfamethoxazole & $35.7 \pm 0.016$ & $63 \pm 0.021$ & $<0.001$ \\
\hline Ampicillin/Sulbactam & $32.1 \pm 0.009$ & $65.2 \pm 0.011$ & $<0.001$ \\
\hline Ciprofloxacin & $32.1 \pm 0.013$ & $48.9 \pm 0.001$ & $<0.001$ \\
\hline Gentamicin & $25.0 \pm 0.019$ & $61.9 \pm 0.014$ & $<0.001$ \\
\hline Piperacillin/Tazobactam & $21.4 \pm 0.015$ & $13.0 \pm 0.018$ & $<0.001$ \\
\hline Cefazolin & $17.8 \pm 0.007$ & $100 \pm 0.010$ & $<0.001$ \\
\hline Amoxicillin-Clavulanic Acid & $14.2 \pm 0.017$ & $30.4 \pm 0.043$ & $<0.001$ \\
\hline Tobramycin & $14.2 \pm 0.011$ & $42.3 \pm 0.023$ & $<0.001$ \\
\hline Ceftazidime & $10.7 \pm 0.040$ & $100 \pm 0.021$ & $<0.001$ \\
\hline Ceftriaxone & $10.7 \pm 0.020$ & $100 \pm 0.011$ & $<0.001$ \\
\hline Cefepime & $10.7 \pm 0.019$ & $100 \pm 0.001$ & $<0.001$ \\
\hline Nitrofurantion & $10.7 \pm 0.013$ & $5.4 \pm 0.001$ & $<0.001$ \\
\hline$\%$ Multidrug resistance & $35.7 \pm 0.031$ & $75 \pm 0.024$ & $<0.001$ \\
\hline
\end{tabular}


Conventional PCR was performed on 30 ESBL positive isolates, $21(70 \%)$ isolates were of E. coli, and $9(30 \%)$ isolates were of $K$. pneumoniae, randomly selected. The SHV enzymes detected only in 11 (36.6\%) isolates. Figure 1 showing the PCR results for 30 samples of both bacteria used in this study, bands of the expected size (768bp) were seen in positive samples. The SHV genotype was observed in $5(55.5 \%)$ of K. pneumoniae isolates and $6(28.5 \%)$ of E.coli isolates (Table 4).

Table 4: Frequency SHV genotype in the ESBL producing E. coli and K. pneumonia.

PCR

$P$ value

\begin{tabular}{lcccc} 
Organism & No. tested & SHV +(\%) & SHV - (\%) & \\
\hline E.coli & 21 & $6(\% 28.5)$ & $15(\% 71.4)$ & 0.159 \\
K. pneumoniae & 9 & $5(\% 55.5)$ & $4(\% 44.4)$ & \\
& & & \\
Total & 30 & $11(\% 36.6)$ & $19(\% 63.3)$
\end{tabular}

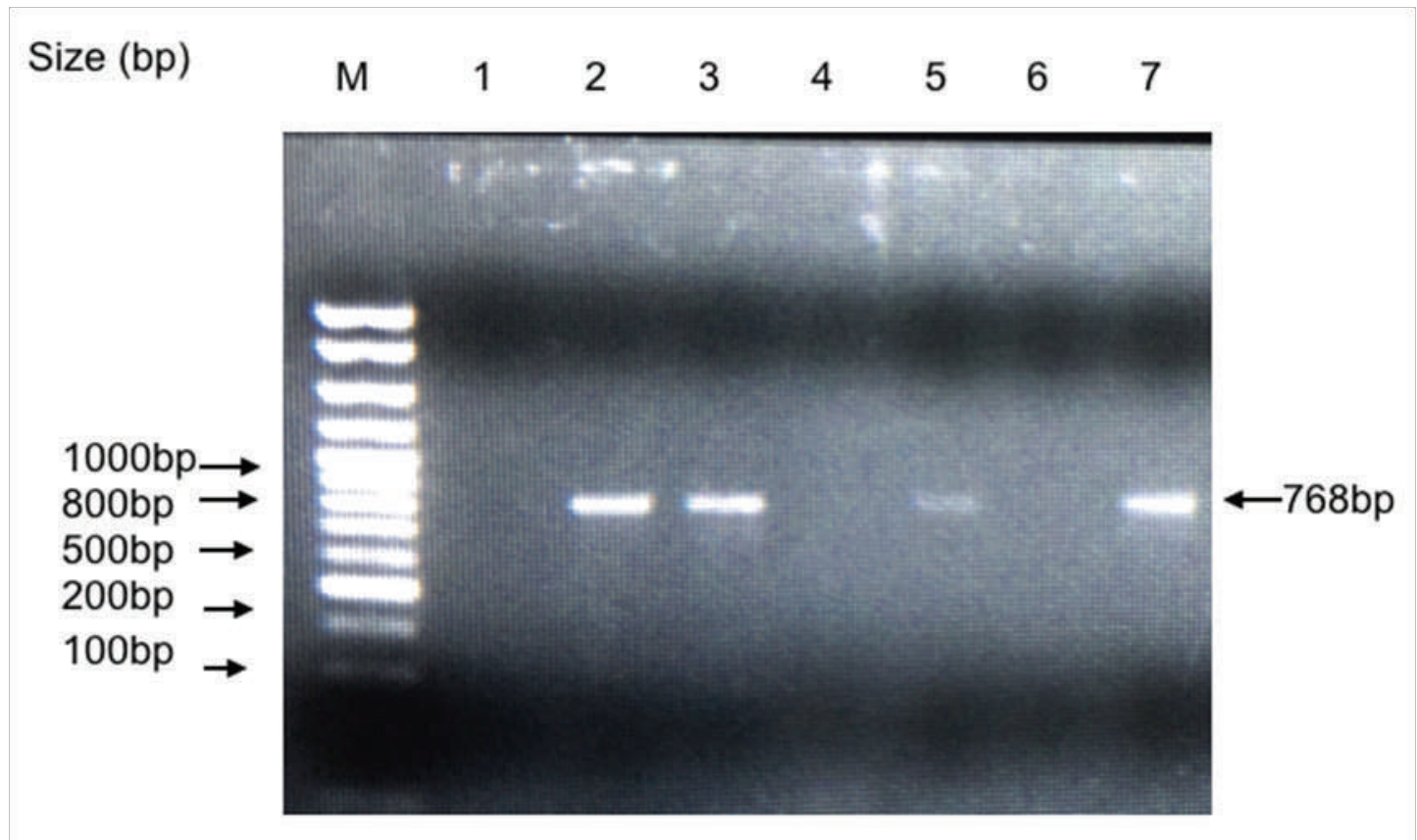

Figure 1: Amplification of bla SHV gene from 7 samples of both $E$. coli and $K$. pneumoniae. Agarose gel electrophoresis of PCR amplicons for the target gene. M: 100-1000bp DNA molecular weight marker, lanes 1-4 are E. coli isolates and lanes 5-7 are K. pneumoniae. 
Regarding antibiotic susceptibility of SHV +ve E. coli and K. pneumoniae isolates, the present study shows that among 11 SHV +veisolates, all were resistant to Ampicillin, Ampicillin/Sulbactam, Trimethoprim/ Sulfamethoxazole, Gentamicin as well asoxyiminocephalosporins (100\%). However, all isolates were susceptible to carbapenems and nitrofurantoin (100\%). The majority of isolates showed a high degree of resistance to Ciprofloxacin with Levofloxacin $(90.9 \%)$ and to Tobramycin (81.8\%) but appeared to be susceptible to Piperacillin/Tazobactam (45.4\%) as shown in Table 5.

\section{Discussion}

The ESBL-producing bacteria have been dramatically spreading worldwide at an alarming rate, and the incidence of ESBL-producing Enterobacteriaceae has threatened the entire world in the current era. At present, the major challenge to infection control is requiring continuous monitoring systems and prevention of the emergence and spread of ESBL-producing Enterobacteriaceae. ${ }^{21}$ The misidentification of ESBLs by diagnostic laboratories could result in wrong therapy via unsuitable prescription of cephalosporins leading to failure in treatment and even causing death. ${ }^{22}$ The accurate detection of ESBL producing microorganisms is a major clinical problem in the laboratories, requiring not only phenotypic tests but also genotypic tests for all genes associated with beta-lactamase production. ${ }^{2}$ The prevalence of $\beta$-lactamase producers and the distribution of ESBL genotypes are different from one year to another and even vary greatly in different geographical areas. ${ }^{23}$ High prevalence of ESBL among K. pneumoniae isolates has been detected in numerous studies. ${ }^{2,21,24,25}$ Similarly in this study, $K$. pneumoniae was the most frequently encountered ESBL-positive isolates (Table 1). From the current study, the overall proportion of ESBL-producing

Table 5: The percentage of SHV +ve $E$.coli and $K$. pneumoniae isolates resistant to the drug tested

\begin{tabular}{lccccccc}
\hline \multirow{2}{*}{ Antibiotics } & \multicolumn{2}{c}{ E.coli } & \multicolumn{2}{c}{ K. pneumoniae } & \multicolumn{2}{c}{ All resistant isolates } \\
& \multicolumn{2}{c}{$\mathbf{n}=\mathbf{6}$} & \multicolumn{2}{c}{$\mathbf{n}=\mathbf{2}$} & \multicolumn{2}{c}{ Total $(\mathbf{n}=\mathbf{1 1})$} \\
& No. & $(\%)$ & No. & $(\%)$ & No. & $(\%)$ \\
\hline Ampicillin & 6 & $(100)$ & 5 & $(100)$ & 11 & $(100)$ \\
Ampicillin/Sulbactam & 6 & $(100)$ & 5 & $(100)$ & 11 & $(100)$ \\
Cefazolin & 6 & $(100)$ & 5 & $(100)$ & 11 & $(100)$ \\
Ceftazidime & 6 & $(100)$ & 5 & $(100)$ & 11 & $(100)$ \\
Ceftriaxone & 6 & $(100)$ & 5 & $(100)$ & 11 & $(100)$ \\
Cefepime & 6 & $(100)$ & 5 & $(100)$ & 11 & $(100)$ \\
Trimethoprim/Sulfamethoxazole & 6 & $(100)$ & 5 & $(100)$ & 11 & $(100)$ \\
Gentamicin & 6 & $(100)$ & 5 & $(100)$ & 11 & $(100)$ \\
Ciprofloxacin & 6 & $(100)$ & 4 & $(80.0)$ & 10 & $(90.9)$ \\
Levofloxacin & 6 & $(100)$ & 4 & $(80.0)$ & 10 & $(90.9)$ \\
Tobramycin & 6 & $(100)$ & 3 & $(60.0)$ & 9 & $(81.8)$ \\
Amoxicillin-Clavulanic acid & 4 & $(66.6)$ & 3 & $(60.0)$ & 7 & $(63.6)$ \\
Piperacillin/Tazobactam & 3 & $(50.0)$ & 2 & $(40.0)$ & 5 & $(45.4)$ \\
\hline
\end{tabular}


$E$. coli and $K$. pneumoniae isolates was $76.6 \%$. A similar finding was reported by other investigators in Iraq $(73.9 \%)^{5}$ In neighboring countries, the prevalence of infection with ESBL-producing Gram-negative bacteria varied from $89.8 \%$ in Iran, ${ }^{26} 31.7 \%$ in Kuwait, ${ }^{27} 54.7 \%-61 \%$ in Turkey, ${ }^{28,29} 50.8 \%$ in Jordan, ${ }^{6} 30.6 \%$ in Saudia Arabia ${ }^{15}$ and $41 \%$ in the United Arab Emirates. ${ }^{30}$ However, our results showed a higher prevalence compared to relative studies from India $(27.6 \%$ $66.7 \%)^{5,31}$ and Kenya $(6 \%)^{2}$ The prime factors for increasing prevalence of ESBLs were attributed to extensive use of thirdgeneration cephalosporins, besides the lack of routine screening for ESBLproducer isolates or isolation guidelines for the infected patients. ${ }^{20}$ Therefore, data produced from this study concerning in terms of these isolates resistance to cephalosporin antibiotics. Faced with the global emergence of antimicrobial resistance, several studies have been undertaken to assess the susceptibility of bacterial pathogens to different antibiotics. $5,12,16$ These findings revealed that there is a widely spreading resistance to most of the available antibiotics, as shown in Table 2. The highest resistance overall was observed against Ampicillin, oxyimino cephalosporin, and ampicillin/ sulbactam, while all isolates showed sensitivity towards carbapenems. In fact, ESBL-producer isolates showed significant higher resistant rate to the $3^{\text {rd }}$ and $4^{\text {th }}$ generation cephalosporin $(100 \%)$ than non ESBL-producer isolates, while these results were comparable with what have been found in published literature..$^{5,16,26}$ The antimicrobial resistance displayed various results. All ESBL-producing E. coli and K.pneumoniae were significantly resistant to ampicillin, 3rd and 4th generations cephalosporins (\%100). These results are consistent with the related studies in the same field., 1,314,23 Furthermore, ESBLproducing isolates were highly resistant to ampicillin/sulbactam (65.2\%), trimethoprim/ sulfamethoxazole $(63 \%)$ and gentamicin
$(61.9 \%)$. These findings might be due to the fact that clinicians and other health care providers may be unaware of the problem of ESBL production by gram-negative bacilli resulting in inappropriate medication. ${ }^{2}$ However all isolates showed sensitivity towards carbapenems $(100 \%)$, The lowest rates of resistance in ESBL-producing isolates were observed for nitrofurantoin (5.4\%), and piperacillin/tazobactam(13\%) data are presented in Table 2. Multi-drug resistant isolates showed to be greater among ESBL-producing isolates than non ESBLproducers. Similar findings were reported in a number of recent studies. ${ }^{2,5,6,26}$ Besides being associated with high morbidity and mortality, the treatment of infections caused by ESBL-producing isolates is becoming increasingly limited. ESBL genotypes were detected in $36.6 \%$ of ESBL-producing isolates of E.coli and $K$. pneumoniae. SHV genes were detected in $55.5 \%$ of $K$. pneumoniae isolates compared to the $28.5 \%$ detection rate within $E$. coli isolates. It is remarkable that SHV type ESBL was frequently found in $K$. pneumoniae. The similar finding reported in other studies. ${ }^{21,32-34}$ Interestingly, ESBLs are mostly detected in K. pneumoniae, as SHV-1 was originally characterized as a plasmid-mediated $\beta$-lactamase, some reports suggest that its production may be native to $K$. pneumoniae since it appears that some clinical $K$. pneumoniae strains encode SHV-1 $\beta$-lactamase production on their chromosomes. ${ }^{22}$ The rate of SHVin this study was a little higher than that reported in a Turkish hospital (24.2\%) all of which were $K$. pneumoniae isolates. ${ }^{32}$ In another study, SHV (92.9\%) was detected as the most prevalent ESBL type in K. pneumoniae isolates. ${ }^{35}$ In Turkey, during 2004 and 2005, SHV was detected in $(46.7 \%)$ E. coli, and K. pneumoniae isolates. ${ }^{36}$ While in a study done in a Saudi Arabian tertiary hospital, $23.1 \%$ of the isolates harbored the SHV gene. ${ }^{16}$ The rate of blaSHV in this study was higher than that reported in an Iranian hospital (5.5\%) 
which were $E$. coli isolates. ${ }^{26}$ Our findings show a lower rate than that reported in Macedonia (49.4\%). ${ }^{33}$ In different studies, the percentage of SHV gene in E. coli and $K$. pneumoniae were determined to be $23.1 \%{ }^{16}, 23.3 \%{ }^{37}$ and $4.3 \%{ }^{6}$ These differences may be caused by several factors such as the type and number of the samples, the number of the isolates studied, genus and species of the isolates. ${ }^{32}$ In our study, a detailed analysis of the 30 isolates of ESBL-producing $E$. coli and $K$. pneumoniae showed that $100 \%$ of SHV +ve isolates of E.coli and $K$. pneumoniae were resistance to betalactam antibiotics, oxyiminocephalosporins, Gentamycin, and Trimethoprim/ Sulfamethoxazole (Table 5). A similar pattern of resistance by Gram-negative pathogens has been reported recently by investigators. ${ }^{2,5,21}$ The highest rate of resistance in SHV +ve isolates was observed for Ciprofloxacin and Levofloxacin (90.9\%). This may be attributed to misuse of Ciprofloxacin and restriction of aminoglycosides in our hospitals. The significantly lower proportion of SHV+ve isolates showing resistance to pepracillin/tazobactam (45.4\%) compared to the amoxicillin/clavulanate resistance rate $(63.6 \%)$, since SHV is more inhibited by tazobactam than clavulanate. The results agreed with a study done in Saudi Arabia. ${ }^{21}$ The extraordinary genetic capacities of microbes have benefited from man's overuse of antibiotics by means of horizontal gene transmission to develop multiple mechanisms of resistance as a result of many years of unremitting selection pressure upon human applications of antibiotics. ${ }^{38}$

\section{Conclusion}

In conclusion, the great difference between ESBL and the spread of clinical isolates of $E$. coli and K. pneumoniae producing these enzymes refer that this is an important health issue in our region. Microbiology laboratories need to be alert to the correct identification and control of infections caused by such microorganisms. The widespread emergence and proliferation of SHV-type ESBLs is particularly noteworthy and may have important implications for clinical microbiology laboratories and physicians treating patients with serious infections caused by these isolates. The most active antimicrobial agent against both ESBL-producers and non ESBLproducer isolates were carbapenem in this study. It is, therefore, strongly recommended to consider carbapenems as the drug of choice for such multi-drug resistant ESBL-producing microorganisms. SHV type of ESBL genes was highly prevalent among $E$. coli and $K$. pneumoniae collected in Erbil city. Therefore, it is necessary to monitor for the spread of these resistance genes and to ensure careful antibiotic use in a hospital setting. Further research should be carried out to study in depth the distribution and evolution of ESBL and other related genes in these pathogens. In addition to that using a larger sample size from various hospitals across the city and different parts of the region to obtain a more representative picture.

\section{Competing interests}

The authors declare that they have no competing interests.

\section{References}

1. Cheong $\mathrm{HT}$, Ho WY, Choo QC, Chew $\mathrm{CH}$. $\beta$-lactamase gene blaSHV detected in bacteria isolated from retail sushi in Kampar, Malaysia. Biomed Res 2014; 25(1):25-31.

2. Juma BW, Kariuki S, Waiyaki PG, Mutugi MM, Bulimo WD.The prevalence of TEM and SHV genes among Extended-Spectrum BetaLactamase-producing Klebsiella pneumoniae and Escherichia coli. Afr J Pharmacol Ther 2016; 5(1):1-7.

3. Harada $\mathrm{Y}$, Morinaga $\mathrm{Y}$, Yamada K, Migiyama $\mathrm{Y}$, Nagaoka $\mathrm{K}$, Uno $\mathrm{N}$, et al. Clinical and Molecular Epidemiology of Extended-Spectrum $\beta$ -lactamase- Producing Klebsiella pneumoniae and Escherichia Coli in a Japanese Tertiary Hospital. J Med Micro Diagn 2013; 2:127.

4. Altayar MA, Thokar MA, Mohammad MA. Extended spectrum B-lactamase-producing Escherichia coli in clinical isolates in Benghazi, Libia: Phenotypic detection and antimicrobial 
susceptibility pattern. Medical Journalof Islamic World Academy of Sciences 2012; 20(2):49-6.

5. Dugal S, Purohit H. Antimicrobial Susceptibility Profile and Detection of Extended Spectrum Beta-Lactamase Production by Gram Negative Uropathogens. Int J Pharm PharmSci 2013; 5(4): 434-8.

6. Al-Mayahie SMG. Phenotypic and genotypic comparison of ESBLproduction by Vaginal Escherichia coli isolates from pregnant and nonpregnant women. Ann Clin Microbiol Antimicrob 2013; $12: 7$.

7. Chaudhary M, Payasi A. Molecular characterization and in vitro susceptibilities of $\beta$-lactamase producing Escherichia coli, Klebsiella species, Acinetobacter baumannii, Pseudomonas aeruginosa and Staphylococcus aureus to CSE1034 and other $\beta$-lactams. Asian Pac J Trop Med 2014; 7(Suppl 1):217-23.

8. Nakamura T, Komatsu M, Yamasaki K, Fukuda S, Miyamoto $\mathrm{Y}$, Higuchi $\mathrm{T}$, et al. Epidemiology of Escherichia coli, Klebsiella Species, and Proteus mirabilis strains producing extended-spectrum beta-lactamases from clinical samples in the Kinki region of Japan. Am J Cli Pathol 2012; 137(4):620-6.

9. Seyedjavadi SS, Goudarzi M, Sabzehali F. Relation between blaTEM, blaSHV and blaCTX-M genes and acute urinary tract infections. J Acute Dis 2016; 5(1):71-6.

10. Sharma M, Pathak S, Srivastava P. Prevalence and antibiogram of Extended Spectrum $\beta$-Lactamase (ESBL) producing Gram negative bacilli and further molecular characterization of ESBL producing Escherichia coli and Klebsiella spp. J Clin Diagn Res $2013 ; 7(10): 2173-7$.

11. Gulfem E. Comparison of conventional method and automatized vitek system in the detection of extended-spectrum beta-lactamase in Escherichia coli and Klebsiella pneumoniae isolates. Afr J Microbiol Res 2013; 7(32):4091- 5.

12. Khanfar HS, Bindayna KM, Senok AC, Botta GA. Extended spectrum beta-lactamases (ESBL) in Escherichia coli and Klebsiella pneumoniae: trends in the hospital and community settings. J Infect Dev Ctries 2009; 3(4):295-9.

13. Gholipour A, Soleimani N, Shokri D, Mobasherizadeh S, Kardi M, Baradaran A. Phenotypic and Molecular Characterization of Extended-Spectrum $\beta$-Lactamase Produced by Escherichia coli, and Klebsiella pneumoniae isolates in an Educational Hospital. Jundishapur J Microbiol 2014; 7(10):e11758.

14. Navon-Venezia S, Hammer-Munz O, Schwartz D, Turner D, Kuzmenko B, Carmeli Y. Occurrence and Phenotypic Characteristics of Extended-Spectrum $\beta$-Lactamases among Members of the Family Enterobacteriaceae at the Tel-Aviv Medical Center (Israel) and Evaluation of Diagnostic Tests. J Clin Microbiol 2003; 41(1):155-8.
15. Dashti AA, West $P$, Paton R, Amyes SGB. Characterization of extended-spectrum b-lactamase (ESBL)-producing Kuwait and UK strains identified by the Vitek system, and subsequent comparison of the Vitek system with other commercial ESBL-testing systems using these strains. J Med Microbiol 2006; 55:417-21.

16. Hassan $\mathrm{H}$, Abdalhamid B. Molecular characterization of extended-spectrum betalactamase producing Enterobacteriaceae in a Saudi Arabian tertiary hospital. J Infect Dev Ctries 2014; 8(3):282-8.

17. Batarseh A, Soneah S, Mardeni R, Elmadni K, Noor M, Abu Ashour N. Antibiotic resistance patterns of multidrug resistance and Extend spectrum beta lactamase producing Escherichia coli urinary isolates at Queen Rani AL-Abdullah hospital for children. Jorden ZUMJ 2013; 19 (5):286-93.

18. Robin F, Delmas J, Schweitzer C, Bonnet R. Evaluation of the Vitek-2 extended-spectrum b-lactamase test against non-duplicate strains of Enterobacteriaceae producing a broad diversity of well-characterized b-lactamases. ClinMicrobiol Infect 2008; 14(2):148-54.

19. Paterson DL, Hujer KM, Hujer AM, Yeiser B, Bonomo MD, Rice LB, et al. Extended-Spectrum beta-Lactamases in Klebsiella pneumoniae. Bloodstream Isolates from Seven Countries: Dominance and Widespread Prevalence of SHV- and CTX-M-Type beta-Lactamases. Antimicrob Agents Chemother 2003; 47(11):3554 -60 .

20. Brin as L, Zarazaga M, S'aenz Y, Ruiz-Larrea F, Torres C. B-Lactamases in Ampicillin-Resistant Escherichia coli Isolates from Foods, Humans, and Healthy Animals. Antimicrob Agents Chemother 2002; 46(10):3156-63.

21. Hassan M, Alkharsah KR, Alzahrani AJ, Obeid OE , Khamis AH, Diab A. Detection of extended spectrum beta-lactamases-producing isolates and effect of AmpC overlapping. J Infect Dev Ctries 2013; 7(8):618-29.

22. Paterson DL, Ko WC, Von GA, Casellas $\mathrm{JM}$, Mulazimoglu L, Klugman KP. Outcome of cephalosporin treatment for serious infections due to apparently susceptible organisms producing extended-spectrum $\beta$-lactamases: Implications for the clinical microbiology laboratory. J Clin Microbiol 2001; 39(6):2206-12.

23. Al-Jasser AM. Extended-spectrum betalactamases (ESBLs): a global problem. Kuwait Med J 2006; 38(3):171-5.

24. Paterson DL, Bonomo RA. Extended-spectrum beta-lactamases: A clinical update. Clin Microbiol Rev 2005; 18:657-86.

25. Tofteland S, Haldorsen B, Dahl KH, Simonsen GS, Steinbakk M,Walsh TR, et al. Effects of phenotype and genotype on methods for detection of extended-spectrum- $\beta$-lactamaseproducing clinical isolates of Escherichia coli 
and Klebsiella pneumoniae in Norway. J Clin Microbiol 2007; 45:199-205.

26. Dallal MS, Sabbaghi A, Aghamirzaeie HM, Lari AR, Eshraghian MR, Mehrabad JF, et al. Prevalence of AmpC and SHV $\beta$-Lactamases in Clinical Isolates of Escherichia coli From Tehran Hospitals. Jundishapur J Microbiol 2013; 6(2):176 -80 .

27. Mokaddas EM, Abdulla AA, Shati S, Rotimi VO. The technical aspects and clinical significance of detecting extended-spectrum beta-lactamaseproducing Enterobacteriaceae at a tertiary-care hospital in Kuwait. J Chemother 2008; 20:44551.

28. Perez F, Endimiani A, Hujer KM, Bonomo RA .The continuing challenge of ESBLs. Curr Opin Pharmacol 2007; 7:459-69.

29. Gur D, Hascelik G, Aydin N, Telli M, Gultekin M, Ogulnc D, et al. Antimicrobial resistance in gram-negative hospital isolates: results of the Turkish HITIT-2 Surveillance Study of 2007. J Chemother 2009; 21:383-9.

30. Al-Zarouni M, Senok A, Rashid F, Al-Jesmi SM, Panigrahi D. Prevalence and antimicrobial susceptibility pattern of extended-spectrum beta-lactamase-producing Enterobacteriaceae in the United Arab Emirates. Med Princ Pract 2008; $17: 32-6$.

31. Hawkey PM. Prevalence and clonality of extended-spectrum beta-lactamases in Asia. Clin Microbiol Infect 2008 ;14 (Suppl 1):159-65.

32. Dagi HT, Al Dulaimi AA, Kus $H$, Seyhan $T$, Findik D, Tuncer I, et al. Genotype distribution of extended Spectrum $\beta$-Lactamase producing Escherichia coli and Klebsiellapneumoniae. Biomed Res 2015; 26(2):235-8.

33. Kaftandzieva A, Trajkovska-Dokic E, Panovski N. Prevalence And Molecular Characterization Of Extended Spectrum Beta-Lactamases (ESBLs) Producing Escherichia coli and Klebsiella pneumoniae. Biol Med Sci 2011; 32:129-41.

34. Bali EB, Acik L, Sultan N. Phenotypic and molecular characterization of SHV, TEM, CTX-M and extended-spectrum $\beta$-lactamase produced by Escherichia coli, Acinetobacterbaumanii and Klebsiella isolates in a Turkish hospital. Afr $\mathrm{J}$ Microbiol Res 2010; 4(8):650-4.

35. Oksuz L, Gurler N. Typing of extended-spectrum betalactamases in Escherichia coli and Klebsiellaspp. strains and analysis of plasmid profiles. MikrobiyolBul 2009; 43:183-94.

36. Gur D, Gulay Z, Akan OA, Aktaş Z, Kayacan CB, Cakici $O$, et al. Resistance to newer beta-lactams and related ESBL types in gram-negative nosocomial isolates in Turkish hospitals: results of the multicentre HITIT study. Mikrobiyol Bul 2008; 42(4):537-44.

37. Fernandes R, Amador P, Oliveira C, Prudêncio C. Molecular Characterization of ESBL -Producing Enterobacteriaceae in Northern Portugal. The Scientific World Journal 2014;
2014:1-6.

38. Nimri L, Azaizeh B. First Report of Multidrug-Resistant ESBL Producing Urinary Escherichia coli in Jordan. British Microbiol Res J 2012; 2(2):71-81. 